\title{
TASEP Speed Process: An Effective Medium Approach
}

\author{
Aanjaneya Kumar and Deepak Dhar \\ Department of Physics, Indian Institute of Science Education and Research Pune
}

\begin{abstract}
We discuss the approximate phenomenological description of the motion of a single second-class particle in a two-species totally asymmetric simple exclusion process (TASEP) on a 1D lattice. Initially, the second class particle is located at the origin and to its left, all sites are occupied with first class particles while to its right, all sites are vacant. Ferrari and Kipnis proved that in any particular realization, the average velocity of the second class particle tends to a constant, but this mean value has a wide variation in different histories. We discuss this phenomena, here called the TASEP Speed Process, in an approximate effective medium description, in which the second class particle moves in a random background of the space-time dependent average density of the first class particles. We do this in three different approximations of increasing accuracy, treating the motion of the second-class particle first as a simple biassed random walk in a continuum Langevin equation, then as a biased Markovian random walk with space and time dependent jump rates, and finally as a Non-Markovian biassed walk with a non-exponential distribution of waiting times between jumps. We find that, when the displacement at time $T$ is $x_{0}$, the conditional expectation of displacement, at time $z T(z>1)$ is $z x_{0}$, and the variance of the displacement only varies as $z(z-1) T$. We extend this approach to describe the trajectories of a tagged particle in the case of a finite lattice, where there are $L$ classes of particles on an $L$-site line, initially placed in the order of increasing class number. Lastly, we discuss a variant of the problem in which the exchanges between adjacent particles happened at rates proportional to the difference in their labels.
\end{abstract}

\section{INTRODUCTION}

There has been a lot of interest in understanding exclusion processes on a line as a simple model of stochastic evolution in systems of interacting particles [1]. These are good models of many physical systems, such as traffic on highways [2], transport in narrow channels [3] and motion of motor proteins on microtubules [4]. Many exact results are known for the simple exclusion process on a line [5]. Several variants of the exclusion process have been studied including multi-species exclusion processes and the partially and totally asymmetric exclusion processes (ASEP and TASEP) $[1,5-8]$.

If we want to study the trajectory of individual particles in an assembly of interacting particles, one often adopts a self-consistent mean-field kind of approximation, in which the motion of the particle occurs in an effective field provided by the others. The best known example being Brownian motion $[9,10]$, that was first studied to describe the motion of pollen grains in a liquid. Other examples of self-consistent treatments include the Hartree-Fock theory of electronic structure of atoms $[11,12]$, and the motion of ions in plasmas in the Vlasov approximation [13].

In this paper, we will discuss this general approach, called the effective medium approach here, in the specific setting of a two-species totally asymmetric exclusion process. We will consider a system of hard-core particles on a 1-dimensional lattice, with two classes of particles. We will consider the evolution from the special initial condition, where there is only one second class particle at the origin, and all sites sites to the left are occupied by first class particles, and all sites to the right of the origin are vacant. The dynamics follows continuous-time Markovian evolution where each first class particle ex- changes position with a second-class particle or vacancy to its right with rate 1 . The second class particle can jump to the left, if forced by a first class particle moving from its left, or jump one space to an empty site on its right, with rate 1 .

For this problem, Ferrari and Kipnis found a rather surprising observation [14]. In their own words, "a second class particle initially added at the origin chooses randomly one of the characteristics with the uniform law on the directions and then moves at constant speed along the chosen one." This is a remarkable property as the system undergoes Markovian evolution, and has no memory. It happens, because if the second-class particle initially, by chance, gets a large positive displacement, in subsequent times it encounters a smaller density of other particles, and hence also moves faster at later times. This is an example of persistence, where time average of one evolution history is very different from ensemble average over all histories of evolution.

While the authors proved this result, they did not discuss how big are the fluctuations in the velocity, and how they decrease with time. In this paper, we will describe this process in a simple Langevin description [15], that also allows us to estimate how the fluctuations in the average speed decrease with time.

We will show that, when the displacement of the second class particle at time $T$ is $x_{0}$, the conditional expectation of displacement, at time $z T(z>1)$ is $z x_{0}$, and the variance of the displacement only varies as $z(z-1) T$. Thus the fluctuations, for fixed $z$, increase as $\sqrt{T}$. Equivalently, we find that if $v^{*}$ is the asymptotic value of velocity of the second class particle, for large $\mathrm{z},\left(v^{*}-x_{0} / T\right)$ has a typical spread of $\frac{1}{\sqrt{T}}$ which goes to 0 as $T$ increases.

The plan of this paper is as follows: in Section II, we define the model precisely. In section III, we discuss the 
description of the trajectory of the second-class particle in a Langevin equation description. We use this to determine the variance of the particle position at time $z T$, given the position at time $T$. In Section IV, we discuss different approximations of increasing accuracy describing the trajectory as a biased random walk on the integer lattice. We then use this approach to study the mean trajectories of a second class particle when the lattice is finite in Section V. We discuss the interesting end-effects that occur in this finite lattice version, explain it using our effective medium approach and outline an interesting new direction that arises through a simple modification in evolution rules. Section VI contains a summary and concluding remarks.

\section{DEFINITION OF THE PROCESS}

We consider a two species TASEP with initial conditions such that a single second class particle is located at the origin of the lattice. To the left of the second class particle, each lattice site is occupied by a first class particle and to its right, each site is vacant. We will denote a first class particle by 1 , a second class particle by 2 and a vacant site by 0 . The allowed nearest neighbor transitions in this process are:

$$
10 \rightarrow 01 ; \quad 20 \rightarrow 02 ; \quad 12 \rightarrow 21
$$

We assume that all these transitions occur with rate 1.

We wish to understand the dynamics of the second class particle in this process. Ferrari and Kipnis proved [14] that the position of the second class particle $X(t)$ at time $t$ follows:

$$
\lim _{t \rightarrow \infty} \frac{X(t)}{t}=U
$$

where $U$ is a uniform random variable on $[-1,1]$.

We will call the process in which the velocity of the second class particle tends to a random number distributed uniformly between $[-1,1]$ as TASEP Speed Process (TSP) and will provide a simple explanation of this remarkable phenomena. The name TSP earlier has been used in the study of joint distribution of the velocities of different particles in a multi-species version of this process [16].

\section{LANGEVIN DESCRIPTION}

We aim to understand the motion of the second class particle, when all the sites to its left are occupied by first class particles and all sites to its right are vacant, using a simple approximation by breaking this problem into two steps:

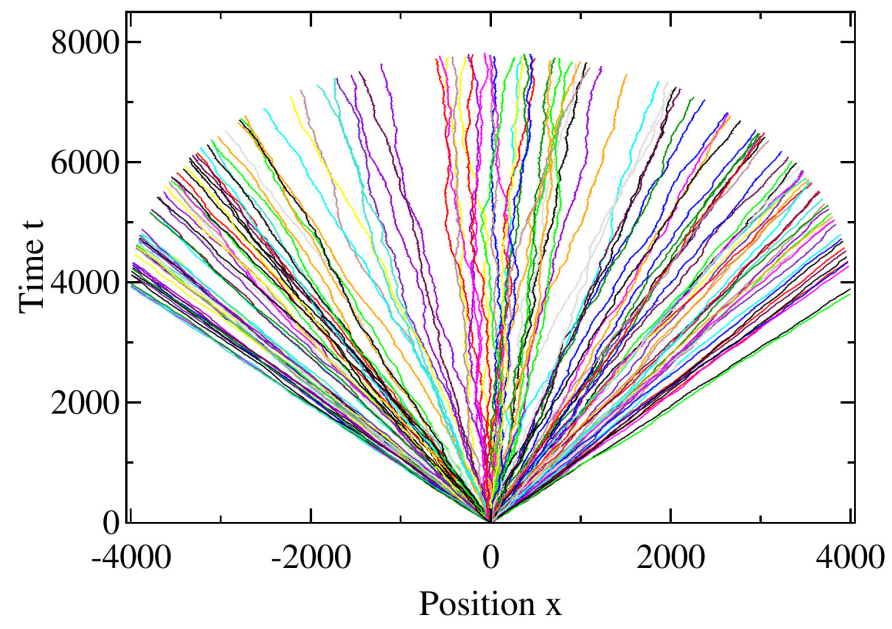

Figure 1. Trajectories of second class particle in the TASEP Speed Process. 150 different trajectories consisting of 4000 steps taken by the second class particle have been plotted.

1. We first discuss how the mean density $\rho(x, t)$ of first class particles evolves in space and time, in the absence of the second-class particle.

2. Then we try to describe the motion of the second class particle moving as a random walk in a spacetime-dependent background field $\rho(x, t)$.

We show that this simple description captures essential features of TSP and allows for further analysis.

Let $x(t)$ denote the position of the second class particle at time $t$. We want to discuss the stochastic properties of this trajectory, by integrating out all the first-class particles. The hydrodynamics of TASEP was first studied by Rost [17]. The coarse-grained evolution of the sea of first class particles in terms of particle density $\rho=\rho(x, t)$ can be described by the partial differential equation:

$$
\frac{\partial \rho}{\partial t}+(1-2 \rho) \frac{\partial \rho}{\partial x}=0
$$

with

$$
\rho(x, t=0)=\theta(-x)
$$

where $\theta(x)$ is the step function, which is 0 for $x<0$, and 1 , for $x>0$. The solution of this partial differential equation is obtained to be:

$$
\begin{array}{rll}
\rho(x, t)=1 & \text { for } & x<-t \\
\rho(x, t)=0 & \text { for } & x>t \\
\rho(x, t)=\frac{1}{2}\left(1-\frac{x}{t}\right) & \text { for } & -t \leq x \leq t
\end{array}
$$

The motion of the second class particle is described by a stochastic differential equation

$$
\frac{d x}{d t}=\bar{V}(\rho(x, t))+\eta(t)
$$


where $\bar{V}$ equals the mean velocity of the particle, and $\eta(t)$ takes into account all the fluctuations away from the mean. By definition, $\langle\eta(t)\rangle=0 . \bar{V}$ is an externally prescribed function of $\rho$ in $\mathrm{Eq}(5)$. In our problem, $\bar{V}=$ $1-2 \rho$, where rho is given by $\mathrm{Eq}(4)$. Hence we write

$$
\bar{V}(\rho(x, t))=1-2 \rho(x, t)
$$

Substituting the value of $\rho(x, t)$ from above:

$$
\frac{d x}{d t}=\frac{x}{t}+\eta(t)
$$

This is a linear differential equation, and may be solved by using an integrating factor. Equivalently, we make a change of variables to $v(t)=x(t) / t$, the mean velocity of the particle. This satisfies the simpler equation

$$
\frac{d v(t)}{d t}=\frac{\eta(t)}{t}
$$

This is easily solved to give

$$
v(z T)-v(T)=\int_{T}^{z T} \frac{\eta\left(t^{\prime}\right)}{t^{\prime}} d t^{\prime}
$$

As $\eta$, by definition has zero mean and $z$ is a real number greater than 1. This gives $\langle v(z T)\rangle=\langle v(T)\rangle$.

We can also determine the variance of $v(T)$ :

$$
\left\langle(v(z T)-v(T))^{2}\right\rangle=\int_{T}^{z T} \int_{T}^{z T} \frac{\left\langle\eta\left(t^{\prime}\right) \eta\left(t^{\prime \prime}\right)>\right.}{t^{\prime} t^{\prime \prime}} d t^{\prime} d t^{\prime \prime}
$$

We expect correlation function $<\eta(t) \eta\left(t^{\prime}\right)>$ to be shortranged. It was shown for TASEP in [18] that correlations $<\rho(x, t) \rho\left(x^{\prime}, t^{\prime}\right)>$ are exponentially decreasing in time $\left|t-t^{\prime}\right|$, unless $x$ and $x^{\prime}$ are such that $x-x^{\prime}=u\left(t-t^{\prime}\right)$, where $u$ is the mean velocity of the flow. In our case, we easily see that while the second-class particle sees a constant density, the mean velocity of first class particles is $1-\rho$, and of second class particle is $1-2 \rho$, and they are not equal. So, in general, the correlation function is short-ranged, and if $D=\int_{-\infty}^{+\infty} d \tau<\eta(t) \eta(t+\tau)>$, we may write $\left.<\eta(t) \eta\left(t^{\prime}\right)>=D \delta\left(t-t^{\prime}\right)\right)$, which gives

$$
<[v(z T)-v(T)]^{2}>=\frac{D(z-1)}{z T}
$$

which goes to 0 as $T$ increases. This shows that the velocity of the second-class particle does get fixed at large $T$.

\section{APPROXIMATE RANDOM WALK DESCRIPTIONS OF THE TRAJECTORY}

While the Langevin description correctly describes the long-time behavior of trajectory correctly, the actual walk occurs on a discrete lattice, and a more accurate description would be as a random walk on a line in continuous time. This we will develop now.

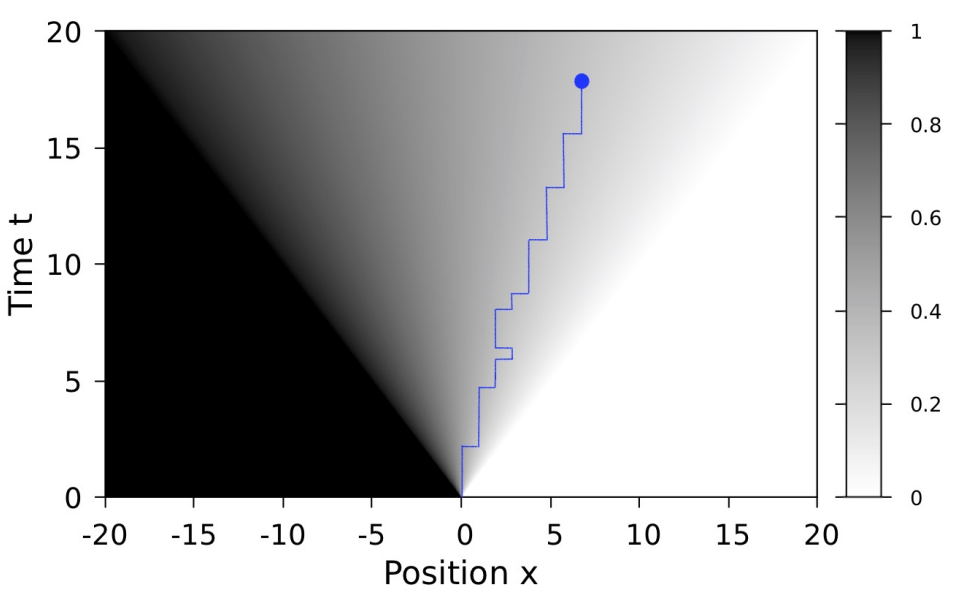

Figure 2. A schematic representing the motion of the second class particle (blue) in the space-time dependent background density plotted as a heat map.

\section{A. Simple Biased Random Walk}

In the spirit of the discussion above, consider motion of a second class particle in uniform density $\rho$. The trajectory then has mean velocity $U=1-2 \rho$, and its time evolution for times $t>>1$ can be discussed as a simple random walk. It is known that if there is no second class particle, then in the steady state, occupation numbers of TASEP have a product measure. Then, in the steady state of TASEP, with a fixed density $\rho$, if we assume that we place a second class particle, with prob. $(1-\rho)$, its site on the right will be empty, and then it jumps with rate 1 . Similarly, with probability $\rho$, the site on its left will be occupied and it will overtake the second class particle with rate 1 . So, we conclude that trajectory of particle is a biased random walk. On a background density $\rho(x, t)$, the second class particle jumps to the left with rate $\rho(x, t)$, and to the right with rate $1-\rho(x, t)$. As a check, the mean velocity is $U=1-2 \rho(x, t)$, which agrees with the exact asymptotic value of velocity [19]. We have simulated this walk on the background $\rho(x, t)$ given by eq. 2-4. The results are shown in Figure 3. We also compare with the the simulation of the original process (Figure 1). We see that while we do get trajectories with velocity fixation, and the velocity $U$ is uniformly distributed in the interval $[-1,1]$, the time taken by the walker to take 4000 steps is roughly the same while, the time taken in TSP shows a clear $\rho$ dependence.

\section{B. Markovian Continuous Time Random Walk}

This shows that the rates of left and right jumps in our simple approximate model do not correctly describe the trajectories of the original problem. The difference occurs because the average density of first class particles near the second-class particle is not the same as in the 


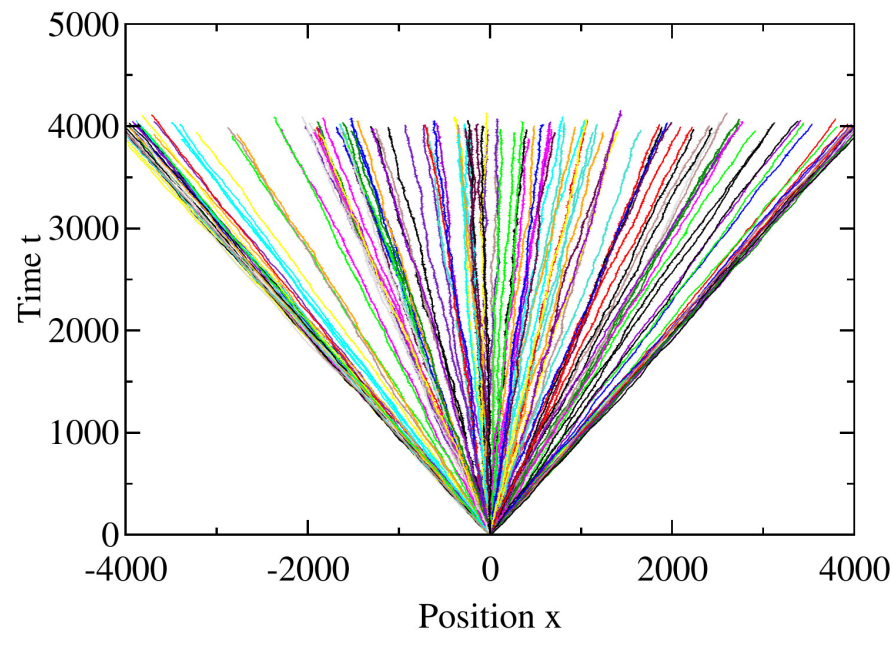

Figure 3. Trajectories of a CTRW with rate $\rho(x, t)$ of jumping to the left and $1-\rho(x, t)$ of jumping to the right. 150 different trajectories consisting of 4000 jumps made by the random walker have been plotted. Notice that the time taken by the walker to take 4000 jumps is roughly the same in each trajectory which is not the case in TSP.

bulk, away from the second class particle. Hence our approximation of using the steady state measure of TASEP to calculate jump rates in the problem with a second class particle is present not adequate. The average density profile near a second class particle in the steady state has been calculated in [20] using the matrix product ansatz. It was shown that the second class particle is attracted to regions with a positive density gradient. More precisely, it was found that for a second class particle on a ring with density $\rho$ of first class particle, in the steady state, the mean density on the site to the right is $2 \rho-\rho^{2}$ and on the site to the left is $\rho^{2}$. This implies the probability of the site to the right being empty is $(1-\rho)^{2}$. If we use a continuous time random walk model with jump rates $(1-\rho)^{2}$ to the right and $\rho^{2}$ to the left we still get mean velocity $=1-2 \rho$. But now the agreement with the simulations is much better as seen in Figure 4.

\section{Continuous Time Random Walk with Waiting Time Distributions}

However, our description is still not sufficiently accurate. If we are given a single long trajectory of the second-class particle, with mean velocity $U$ in the original process between times $T$ and $n T$, for $T>>1$, and also one generated using the Markovian jump rates descibed above, can one distinguish between them? The answer is yes. Clearly, in the Markovian approximation, the waiting times between successive jumps are independent random variables, with a distribution that is a simple exponential. One can easily verify that in the original process the waiting time intervals do not have an exponential distribution. This comes from the fact that since

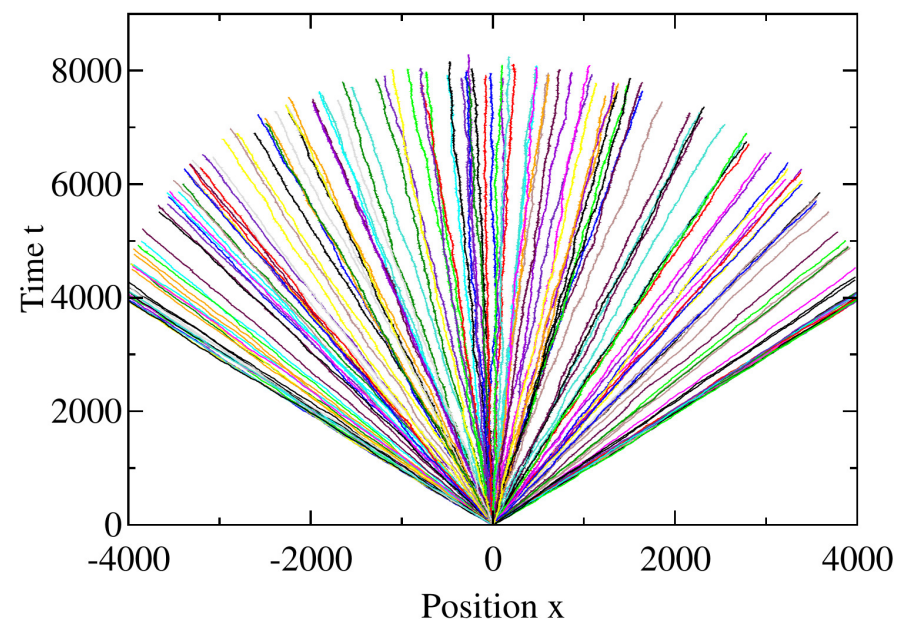

Figure 4. Trajectories of second class particle in the CTRW model with jump rates $(1-\rho(x, t))^{2}$ to the right and $(\rho(x, t))^{2}$ to the left. 150 different trajectories consisting of 4000 steps taken by the second class particle have been plotted.

occupancy of neighbors by first class particles have nontrivial correlations in time, so the probabilities of jump in nearby time intervals $\left[t, t+\Delta t_{1}\right]$ and $\left[t+\Delta t_{1}, t+\Delta t_{1}+\Delta t_{2}\right]$ are not uncorrelated.

The trajectories in the TASEP Speed Process show a nearly exponential distribution of waiting times only for velocities close to 1 and -1 whereas a clear departure from the exponential distributiom is observed for smaller velocities. Figure 5 shows the distribution of waiting times for the TSP and the CTRW model with jump rates. The histograms were plotted for two trajectories, consisting of 8000 steps taken by the second class particle, of velocity (a) 1 and (b) 0.2 . It is clearly seen that an even more accurate modelling of the trajectory will be as a random walk which involve a continuous time non-Markovian walk with a prescribed distribution of residence times $f(\tau)$, with probability to jump left or right given by $p(\tau)$ and $1-p(\tau)$. The calculation of the exact functions $f(\tau)$ and $p(\tau)$ is rather difficult, and will not be attempted here. We can take these to be approximately determined from simulations.

Of course, even this modelling of the trajectory as a continuous time random with a distribution of waiting times is approximate. In the original process, the waiting times between successive jumps are only approximately uncorrelated. But going beyond this description falls outside our aim of providing a simple approximate description of the trajectories.

\section{EXTENSIONS OF OUR APPROACH}

This work can be extended to the case of a multispecies partially asymmetric exclusion process (ASEP). It was conjectured in [17] that even in partially asymmetric case, the asymptotic velocity tends to a uniformly 


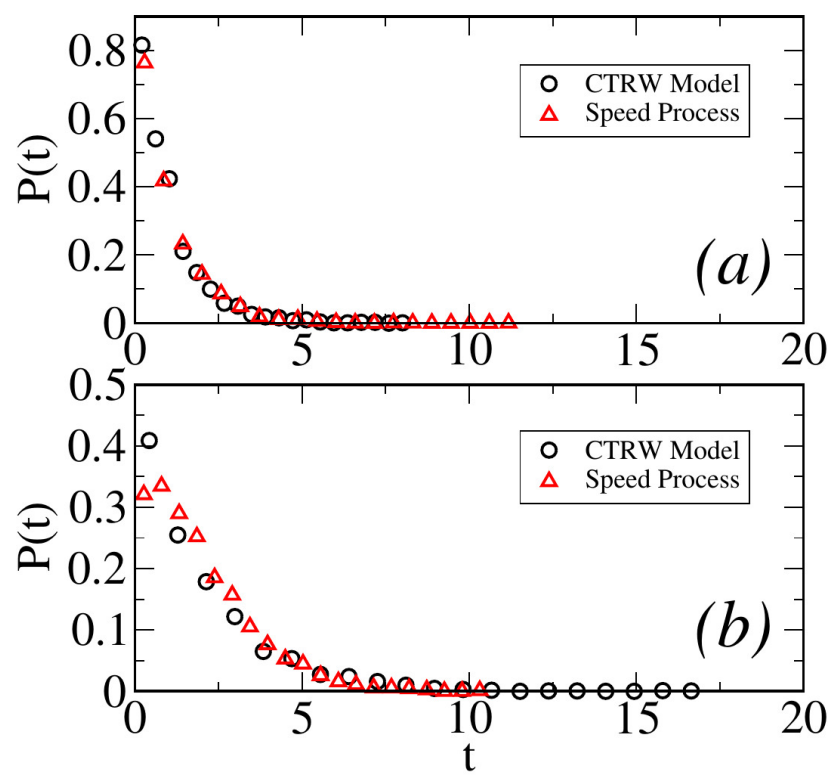

Figure 5. Waiting time distributions for trajectories with speeds (a) 1 and (b) 0.2 in the CTRW model (black circle) and the TASEP Speed Process (red circle). For speeds close to 1 , the waiting time distribution for the TASEP Speed Process matches closely with the exponential waiting times of the CTRW model. However, for intermediate speeds, a clear departure from exponential waiting time distribution is observed.

distributed random variable. More precisely, in this case, if we start with the initial conditions as before and look at the motion of the second class particle, then:

$$
\lim _{t \rightarrow \infty} \frac{X(t)}{t}=U_{p}
$$

where $X(t)$ is the position of the second class particle at time $t$ and $U_{p}$ is a uniform random variable between $[-(2 p-1),(2 p-1)]$ where $p$ is the rate of jumping to the right $(1-p$ being the rate of jumping to the left). An Langevin description can be developed for this as the evolution of density for first class particles is given by:

$$
\frac{\partial \rho(x, t)}{\partial t}+(2 p-1)(1-2 \rho(x, t)) \frac{\partial \rho(x, t)}{\partial x}=0
$$

Even in the case of multi-species ASEP, our analysis goes through and the fluctuations about the average velocity die out as $t^{-1 / 2}$.

The finite lattice version of the multi-species problem [21] offers an interesting extension to the effective medium approach. The system considered is a finite lattice with $n$ sites in which, each site is occupied by a particle and its class is labeled by its initial position on the lattice. The time evolution of the system is given by the stochastic nearest neighbor exchange rule:

$$
i j \stackrel{\text { rate } 1}{\longrightarrow} j i \quad \text { for all } i<j
$$

If we wish to study the dynamics of a tagged particle of the $k$-th class, it is clear that the problem is, again, reducible to a two species problem with particles of $l$-th class $(l<k)$ being equivalent to first class particles, particles of $m$-th class $(m>k)$ being holes and the tagged particle being the second class particle.

The motion of a tagged particle is strongly affected by the ends and displays an interesting behaviour - initially, its dynamics of the tagged particle mimics the dynamics of a tagged particle in TSP on an infinite line. However, at later times, the particle reaches a growing impenetrable region of density 1 (0) on the right (left) and travels along with it, remaining at the moving end of this region at subsequent times, till its absorbing position. This is expected as the lattice is finite and after some time, clearly the first class particles (holes) start to get accumulated at the right (left) boundary. It is interesting to note that the absorbing position of a particle whose initial position was $k$ is always $n-k$. This behaviour can be described by CTRW model with jump rates given by the following background density:

$$
\rho(x, t)=\begin{array}{ccc}
0 & \text { for } & x \leq l(t) \\
1 & \text { for } & x \geq n-r(t) \\
\frac{1}{2}\left(1-\frac{x-k}{t}\right) & \text { for } & -t+l(t)<x-k<t-r(t) \\
1 & \text { for } & l(t) \leq x<k-t+l(t) \\
0 & \text { for } & k+t-r(t) \leq x<n-r(t)
\end{array}
$$

$l(t)$ and $r(t)$ are the mean widths of impenetrable regions of density 1 and 0 on the right and left boundary respectively and they satisfy:

$$
r(t)=\begin{array}{clc}
0 & \text { for } & t \leq(n-k) \\
t+(n-k)-2 \sqrt{t(n-k)} & \text { for } t_{s} \geq t>(n-k) \\
k & \text { for } & t>t_{s} \\
0 & \text { for } & t \leq k \\
t+k-2 \sqrt{t k} & \text { for } & t_{s} \geq t>k \\
n-k & \text { for } & t>t_{s}
\end{array}
$$

where $t_{s}$ is the mean time taken by the tagged particle to reach its absorbing position and is given by $t_{s}=n+$ $2 \sqrt{k(n-k)}$.

We simulated the process on a lattice of 1000 sites and looked at the various trajectories of the particle labelled 200. Figure 6 shows 150 such trajectories and Figure 7 shows analogous results for the effective medium description using a continuous time random walker on a line with jump rates determined by the background density given in $\mathrm{Eq}(13)$. 


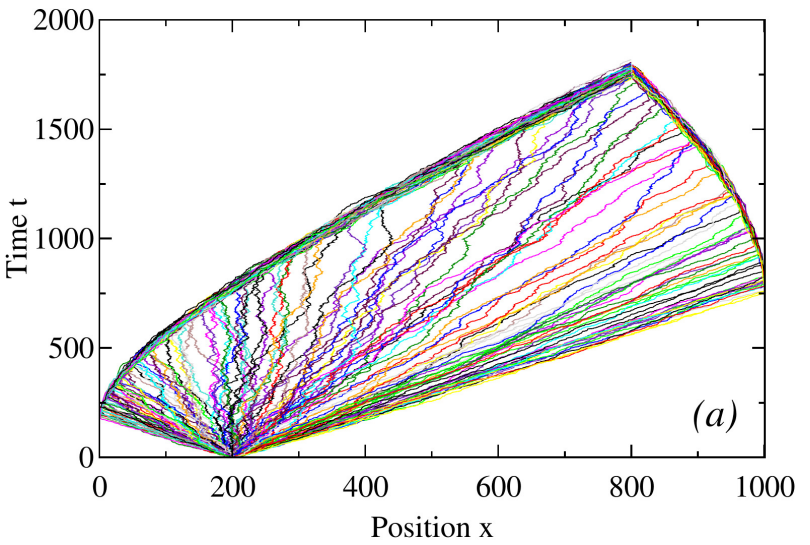

Figure 6. 150 different trajectories of the particle labelled 200 in the finite lattice version of TSP with 1000 particles.

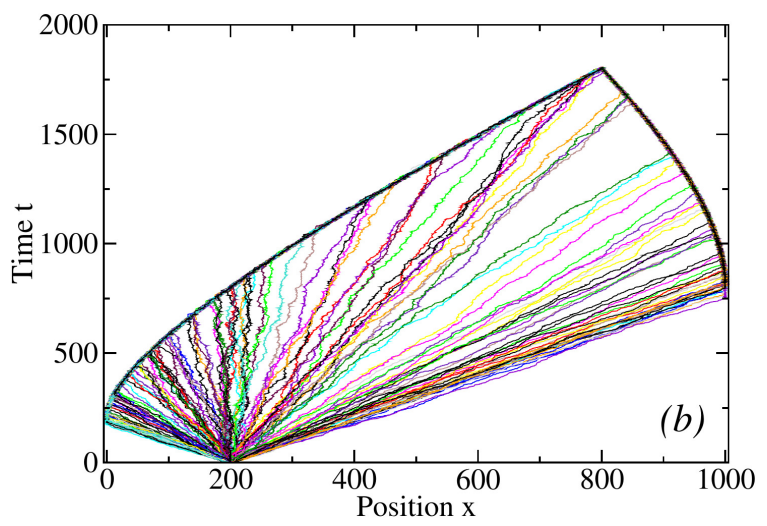

Figure 7. 150 different trajectories of a continuous time random walker with space time dependent jump rates determined by the evolving density of first class particles defined by $\operatorname{Eq}(13)$.

As an interesting variation to the multi-species problem, consider a 1D lattice where each lattice site is occupied with a particle and the class of each particle is labeled by its position on the lattice with only the following nearest neighbor transitions allowed:

$$
\text { ij } \stackrel{\text { rate }(j-i)^{\alpha}}{\longrightarrow} j i \quad \text { for all } i<j
$$

This model is clearly a better model for traffic flow if we visualize the $x$-coordinate of particles to not be their position in real space, but their relative order on the road as the overtakings between two particles happen at rates proportional to the difference between their labels (which is a proxy for the velocity).

Some special cases of this model have been studied before $[6-8]$. It is known that the steady state of such a model on a 1D lattice with open boundary conditions and $\alpha=1$, in which there was an additional feature that particles could enter the system from the left end and leave from the right at rates depending on their labels, can be obtained by a matrix product ansatz. This was

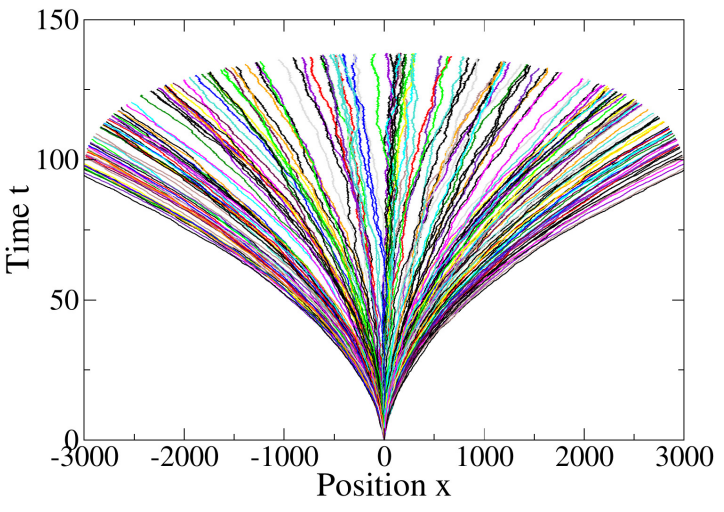

Figure 8. 150 trajectories consisting of 3000 jumps made by a tagged particle in the modified multi-species exclusion process with $\alpha=0.5$.

later generalized to obtain the steady state properties of this system on a ring.

We consider the dynamics of a tagged particle in this modified multi-species exclusion process on an infinite line for a general $\alpha$ where particles do not enter or exit the system. This problem cannot be reduced simply to the 2 -species problems as each particle interacts with every other particles differently. However, we find that something analogous to the "velocity selection" in TSP happens in this process as well. The trajectories of the tagged particle in this process seem to belong to the family of curves $t=a x^{1-\alpha}$ for $\alpha \neq 1$ and $t=\ln a x$ for $\alpha=1$ where $a$ parametrizes the trajectories. A heuristic argument for this is as follows:if a particle has moved distance $x$ in time $t$, then the typical change in velocity it encounters with its neighbor is proportional to $\mathrm{x}$. Then $d x / d t \sim x^{\alpha}$ implies that $x \sim t^{1 /(1-\alpha)}$. for $\alpha \neq 1$ and $t \sim \ln x$ for $\alpha=1$.

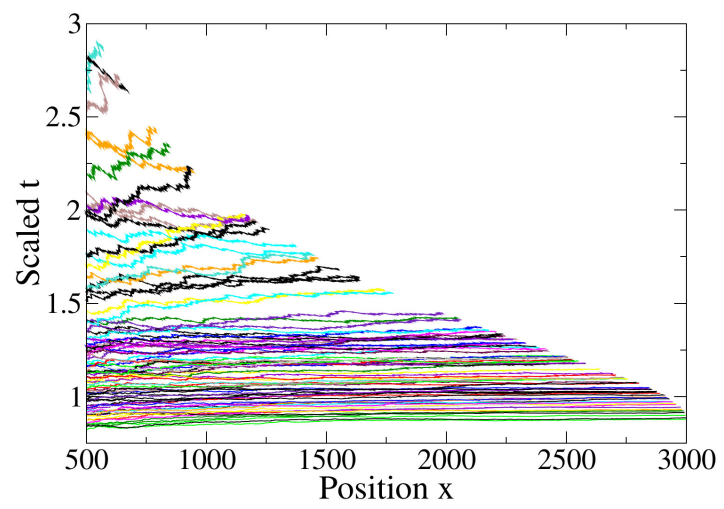

Figure 9. Trajectories of the tagged particle in the modified multi-species exclusion process with $\alpha=0.5$ after time coordinate $t$ is scaled as $\frac{t}{2 \sqrt{x}}$. 150 different trajectories are plotted showing that all the trajectories belong to a family of curves given by $t \sim \sqrt{x}$.

We demonstrate numerical results of our scaling in Figures 8 and 9 for $\alpha=\frac{1}{2}$. Figure 8 shows 150 different 
space-time trajectories of the process and Figure 9 shows the trajectories when the time coordinate $t$ is scaled as $\frac{t}{2 \sqrt{x}}$ where we have only chosen the trajectories whose displacement always remains positive. We see that $t / \sqrt{x}$ is nearly constant for each trajectory, but different trajectories have very different values of this variable. Finding the distribution of the asymptotic value of $a$ over different trajectories remains an open problem.

\section{SUMMARY AND CONCLUDING REMARKS}

In summary, we discussed the effective medium approach to describe the motion of a tagged particle in the time-evolving background other particles. We provided a simple Langevin description of the dynamics, that captures the key features of the large-scale behavior of TSP, and also calculated the variance of the average velocity within one history, and for different histories. We discussed how to improve the effective medium description to take into account different additional features of the trajectories. These were approximating the trajectory as a biassed random walk, with rates of walk calculated from the steady state of the TASEP, in the absence of the second-class particle. We found that to get a quanti- tative agreement with the original process, one has to incorporate the modification of the average density profile that occurs near the second-class particle. Also, while the time evolution of the original process was Markovian by definition, the evolution of the projected process is non-Markovian. This is most easily seen in the nonexponential distribution of waiting times between jumps in the motion of the second-class particle. We proposed a non-Markovian continuous time random walk with a distribution of waiting times between jumps as a good description of this. We later extended our approach to a finite lattice version of the TSP and studied the trajectories of a tagged particle in this process. In this case, interesting end-effects are seen which we explained using the effective medium approach. Lastly, we looked at a modified multi-species exclusion process in which exchanges between adjacent particles happened at rates proportional to the difference in their labels. We showed that in this process too, the motion of a tagged particle shows a behaviour in which it initially "chooses" a trajectory from a family of curves and sticks to it asymptotically. A better understanding of our heuristic arguments, and numerical observations about this process for a general $\alpha$ seems to be an interesting problem for further study.
[1] K. Mallick, Physica A: Statistical Mechanics and Its Applications 418, 17 (2015).

[2] D. Chowdhury, L. Santen, and A. Schadschneider, Physics Reports 329, 199 (2000).

[3] O. Bénichou, P. Illien, G. Oshanin, A. Sarracino, and R. Voituriez, Journal of Physics: Condensed Matter 30, 443001 (2018).

[4] T. Chou, K. Mallick, and R. K. P. Zia, Reports on Progress in Physics 74, 116601 (2011).

[5] P. L. Krapivsky, S. Redner, and E. Ben-Naim, A Kinetic View of Statistical Physics (Cambridge University Press, 2010).

[6] S. Das, D. Dhar, and S. Sabhapandit, Physical Review E 98, (2018).

[7] V. Karimipour, EPL 47, 304 (1999).

[8] V. Karimipour, Phys. Rev. E 59, 205 (1999).

[9] N. G. V. Kampen, Stochastic Processes in Physics and Chemistry (Elsevier, 1992).

[10] B. Duplantier, in Einstein, 1905-2005: Poincaré Seminar 2005, edited by T. Damour, O. Darrigol, B. Duplantier, and V. Rivasseau (Birkhäuser Basel, Basel, 2006), pp. 201-293
[11] P. Echenique and J. L. Alonso, Molecular Physics 105, 3057 (2007).

[12] M. Schwartz and E. Katzav, J. Stat. Mech. 2008, P04023 (2008).

[13] F. Pegoraro, F. Califano, G. Manfredi, and P. J. Morrison, Eur. Phys. J. D 69, 68 (2015).

[14] P. A. Ferrari and C. Kipnis, Annales de l'I.H.P. Probabilités et Statistiques 31, 143 (1995).

[15] R. Livi and P. Politi, Cambridge Core (2017).

[16] G. Amir, O. Angel, and B. Valkó, Ann. Probab. 39, 1205 (2011).

[17] H. Rost, Z. Wahrscheinlichkeitstheorie Verw Gebiete 58, 41 (1981).

[18] S. N. Majumdar and M. Barma, Phys. Rev. B 44, 5306 (1991).

[19] P. A. Ferrari, Probab. Th. Rel. Fields 91, 81 (1992).

[20] B. Derrida, S. A. Janowsky, J. L. Lebowitz, and E. R. Speer, J Stat Phys 73, 813 (1993).

[21] O. Angel, A. Holroyd, and D. Romik, Ann. Probab. 37, 1970 (2009). 